\title{
Importance of avoiding surgery delays after initial discovery of suspected non-small-cell lung cancer in clinical stage IA patients
}

This article was published in the following Dove Press journal: Cancer Management and Research

\author{
Hiroaki Kuroda' \\ Yusuke Sugita' \\ Yuko Ohya ${ }^{2}$ \\ Tatsuya Yoshida ${ }^{2}$ \\ Takaaki Arimura' \\ Noriaki Sakakura' \\ Toyoaki Hida ${ }^{2}$ \\ Yasushi Yatabe ${ }^{3}$ \\ Yukinori Sakao' \\ 'Department of Thoracic Surgery, \\ Aichi Cancer Center Hospital, \\ Nagoya, Japan; ${ }^{2}$ Department of \\ Thoracic Oncology, Aichi Cancer \\ Center Hospital, Nagoya, Japan; \\ ${ }^{3}$ Department of Pathology and \\ Molecular Diagnostics, Aichi Cancer \\ Center Hospital, Nagoya, Japan
}

Correspondence: Hiroaki Kuroda Department of Thoracic Surgery, Aichi Cancer Center Hospital, I-I, Kanokoden, Chikusa-ku, Nagoya 464-868I, Japan

Tel +8I 527626 III

Fax +8I 527635233

Email h-kuroda@aichi-cc.jp
Introduction: The natural history of consolidation on computed tomography (CT) rarely includes invasive cancers, and evidence of the ideal timing for surgical intervention via longterm follow-up studies remains unknown.

Methods: Between January 2012 and June 2017, pulmonary resection was undertaken in 293 clinical IA patients who were followed-up for $>6$ months after the first detection of potential non-small-cell lung cancer (NSCLC) opacities. We evaluated the corresponding HRs and compared the recurrence risk with the CT follow-up duration.

Results: HRs calculated for the longest intervals were compared between two patient subsets: a shorter-interval surgery group (SISG: $41.3 \%$; mean follow-up interval, $13.5 \pm 5.3$ months) and a longer-interval surgery group (58.7\%; mean follow-up interval, $54.9 \pm 25.6$ months). On Cox multivariate regression analyses, CT consolidation (ratio $>0.5$ ), an abnormal carcinoembryonic antigen and a triple-negative mutation showed an independent association with an unfavorable prognosis, as measured by disease-free survival after the first detection of potential NSCLC opacities. The longer-interval surgery group fared significantly better than the SISG in terms of 5-year overall survival after the first detection $(99.3 \%$ vs $93.1 \%, P<0.01)$; the 3 -year overall survival after the first detection was significantly shorter in the high-risk SISG (presence of two factors from the three) than that in the low-risk SISG (presence of 0 or one factor; $100 \%$ vs $73.3 \%, P<0.01$ ). Conclusion: Our study indicates that the patients with potential NSCLC opacities who are able to wait for more than 2 years prior to pulmonary resection may be likely to have a favorable prognosis, whereas early judgment for surgical resection should be required for avoiding surgical delays.

Keywords: non-small-cell lung cancer, computed tomography, natural history, surgery delay, consolidation, ground glass opacity

\section{Introduction}

Several radiological studies have shown that the persistence of a solid component is a strong biological marker of lung cancer in patients with ground glass opacities (GGOs) and that such patients should be closely monitored. ${ }^{1}$ The solid component, which typically appears as a consolidation on computed tomographic (CT) images, may be compatible with a fibrotic focus, alveolar collapse, or cancer proliferation. ${ }^{2}$ The International Early Lung Cancer Action Project (IELCAP) has cited a $63 \%$ rate of malignancy for part-solid nodules, as compared to rates of $18 \%$ and $7 \%$ for nonsolid and solid nodules, respectively. ${ }^{3}$ Hasegawa et al have also reported a mean volume doubling time of 813, 457, and 149 days for nonsolid, part-solid, and solid nodules, respectively. ${ }^{4}$ Considering this natural tendency for slow growth, a 5-year follow-up period may be inadequate for conclusive assessments. Moreover, it is essential that a 
pathological diagnosis be obtained to aid in the investigation of GGO behavior.

Results of the National Lung Screening Trial (NLST) suggest that some of these part solid nodules may evolve into aggressive lung cancer over time. Their changing appearance, which for now remains the best biomarker for cancer progression, has been documented in CT scans. Their findings add further support to a conservative approach of annual follow-up visits for the management and treatment of partsolid nodules (especially if solitary), based on prospective analyses of lung cancer deaths. ${ }^{5-7}$ The clinical significance of CT consolidation is broadly accepted, and pertinent procedures have been proposed accordingly by many sources. ${ }^{8}$ Although the threshold for subjecting growing GGOs to pulmonary resection is extremely critical, and much is currently known of the timing of transversion, underestimates of CT consolidation occur to no small extent. Management decisions may then be challenging, and there is a point at which pulmonary resection should not be delayed in order to achieve better long-term patient outcomes.

The aim of this study was to explore factors that impact on follow-up protocols in this setting and to assess the need for prompt treatment (including pulmonary resection) by analyzing patients with histologically proven non-small-cell lung cancer (NSCLC) who were followed-up for $>6$ months and were preoperatively diagnosed with clinically invasive adenocarcinoma (cIA), and to settle the question, "When is the surgical intervention necessary during the natural history of potential NSCLC opacities?"

\section{Materials and methods}

In this retrospective review, we examined 293 patients (men, 152; women, 141) with primary NSCLC diagnosed with cIA, who had undergone pulmonary resection $>6$ months after the first detection of potential NSCLC opacities on CT. The surgeries were performed between January 2012 and June 2017 at the Aichi Cancer Hospital. All patients provided written informed consent for pulmonary resection and for anonymous participation in the study. Clinical data for staging were generated through CT images of the chest and abdomen, magnetic resonance imaging of the head, bone scintigraphy, and/or positron emission tomography. Tumors were staged using the TNM classification system (eighth edition); pathological findings were interpreted according to the 2004 World Health Organization terminology., 9,10

We calculated HRs for appropriate times to cease followup observations and proceed to pulmonary resection and compared the recurrence risk with the duration of follow-up
CT observations. For this study, CT consolidation on thin CT (10-20 mm thickness) slices served as a critical radiological measure. Data collected from patient records included age, gender, preoperative serum carcinoembryonic antigen (CEA) levels, smoking status (pack-years), history of malignancy, pathological TNM stage, and mutation status (EGFR/ALK/ $K R A S)$. This study was conducted in accordance with the Declaration of Helsinki. Since the anonymity of individual patients was ensured, the institutional review board of Aichi Cancer approved this study (2017-1-295), provided that preoperative informed consent was obtained in each instance. All patient records and information were anonymized prior to analysis.

\section{The indications of surgical timing at our department}

The following indications are usually required for the decision of pulmonary resection.

- When patient request and consent are obtained.

- When maximum tumor size is more than $15 \mathrm{~mm}$ on lung setting on $\mathrm{CT}$.

- When consolidation (the solid component on GGO) on lung setting on CT is appeared, even if tumor size is less than $15 \mathrm{~mm}$.

- When there is the shadow appearance on mediastinal setting on CT with an indistinct consolidation.

We defined this maximum size on mediastinal setting on CT as mediastinal diameter (MD).

\section{Statistical analysis}

All computations relied on standard software (SPSS version 17.0; SPSS Inc. [IBM Corporation], Chicago, IL, USA). Comparisons between the two groups were performed by Mann-Whitney $U$-tests. The Kaplan-Meier method was used to analyze survival rates in the patient subsets; between-group differences in survival were assessed with the log-rank test. Potential correlates of survival were subjected to univariate and multivariate analyses using the Cox proportional hazards regression model. HRs and median survival rates are presented with $95 \%$ CIs. Statistical significance was set at $P<0.05$.

\section{Results \\ Detection of surgical timing}

During the study reference period, 293 patients with cIA who were followed-up for $>6$ months prior to surgical resection were eligible for inclusion in this study. Sublobar resection was performed in $54.9 \%$ (161/293) patients, while $45.1 \%$ 
(132/293) patients underwent more than lobectomy, including one pneumonectomy. However, one patient $(0.3 \%)$ was eventually diagnosed with pathological stage M1A (dissemination). A histogram showing the time elapsed between the first detection of potential NSCLC opacity on CT and the date of surgery is shown in Figure 1 (median interval, 29 months [ 2 years]). An adjusted graph of the association between disease-free survival (DFS) at the first CT detection and the time interval from detection to surgical resection is shown in Figure 2A. When comparing HRs of the longest

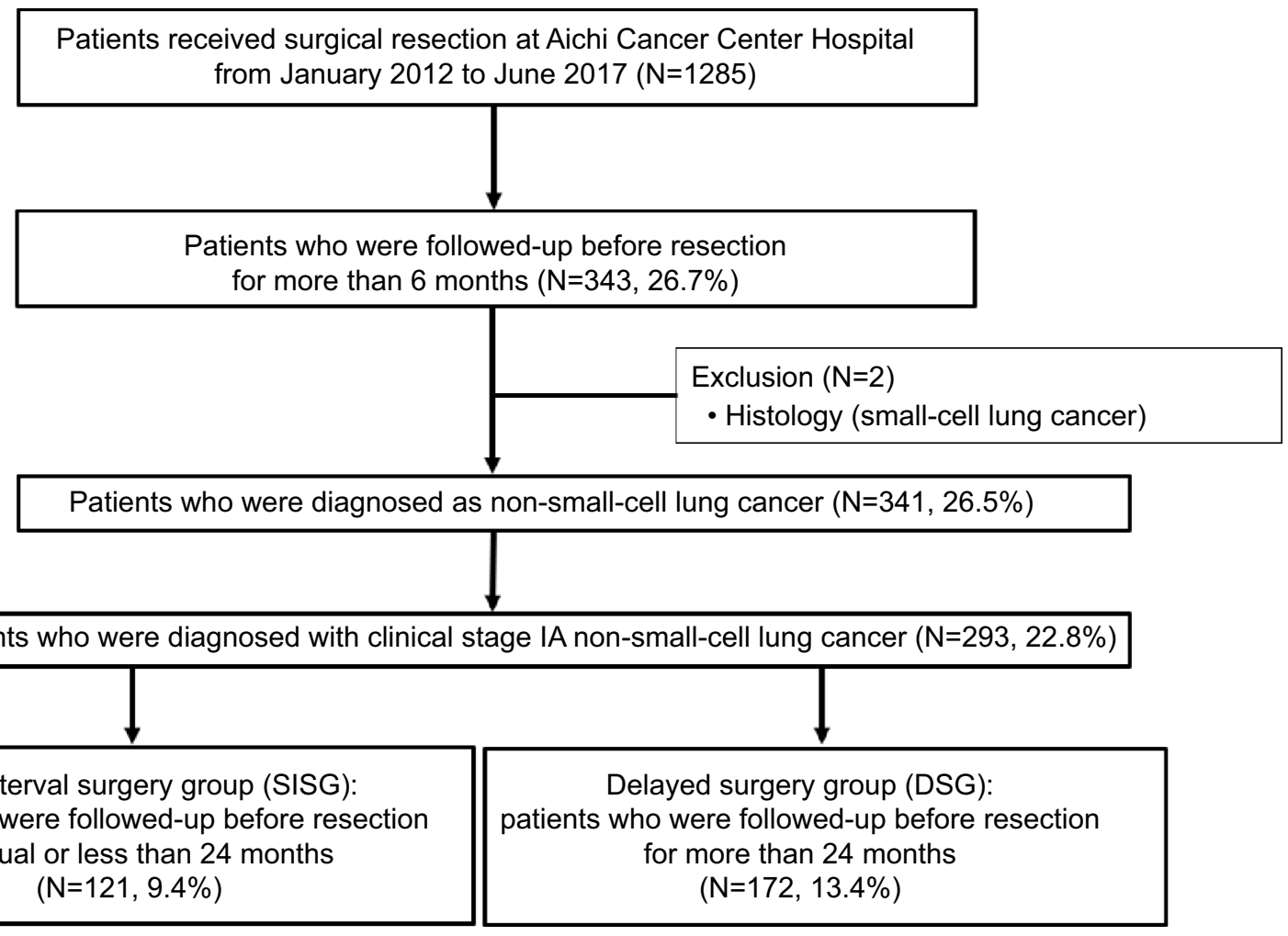

Figure I Schematic illustration of the inclusion criteria for this study.

A

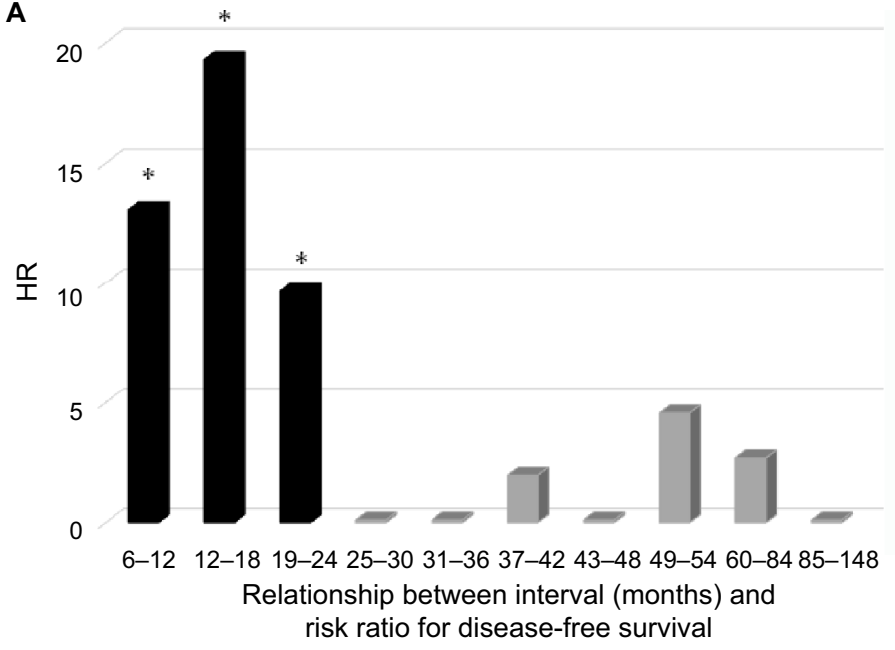

Figure 2 Histogram of enrolled patients and the therapeutic outcomes.

Notes: (A) The therapeutic prognosis corresponded to disease-free survival based on the longest intervals ( $>84$ months [6 years]). Black bars: HR of overall survival in each pathological size with significance $* P<0.05$; gray bars: non-significant. (B) The time period between the first detection of suspected non-small-cell lung cancer opacities and the date of pulmonary resection. Black bars: shorter-interval surgery group (SISG) and gray bars: longer-interval surgery group (LILG).
B

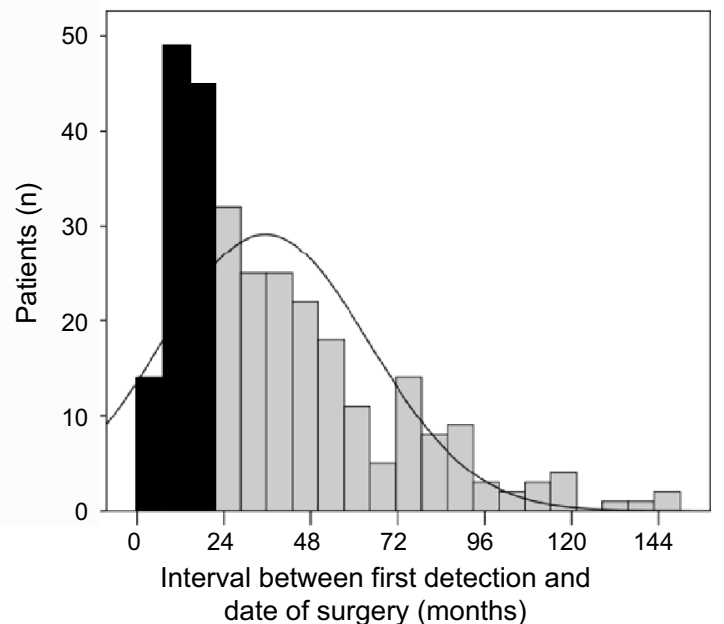


intervals (>84 months), DFS declined gradually as intervals grew longer. By comparison, DFS at shorter intervals $(\leq 24$ months) differed significantly (Figure 2A). In this regard, two patient subsets were established: the shorter-interval surgery group (SISG: 121/293, 41.3\%; mean follow-up interval, $13.5 \pm 5.3$ months) and the longer-interval surgery group (LISG: 172/293, 58.7\%; mean follow-up interval, 54.9 \pm 25.6 months; Figure 2B).

\section{Patient characteristics in each group}

Characteristics of the study population are summarized in Table 1. Exposure to tobacco (pack-years) was significantly higher in members of the SISG (vs the LISG); however, the history of malignancy in the SISG was significantly lower than that in the LISG. The two groups did not show any significant differences with respect to surgical approach and procedure (thoracotomy vs thoracoscopy; $P=0.51$; and sublobar resection vs lobectomy; $P=0.90$; respectively). The diagnoses of adenocarcinoma in situ (AIS) or minimally IA (MIA) in the LISG were not significantly more frequent than those in the SISG $(33.7 \%$ vs $24.0 \%$, respectively; $P=0.07$ ); however, the LISG displayed a higher rate of equal to or more than pathological stage IIA disease $2-4(90.7 \%$ vs $79.3 \%$, respectively; $P<0.01)$. No significant between-group difference was observed with respect to mutations $(P=0.26)$.

\section{Patient outcomes}

The 5-year DFS and overall survival (OS) rates after the first CT detection in the LISG were significantly longer

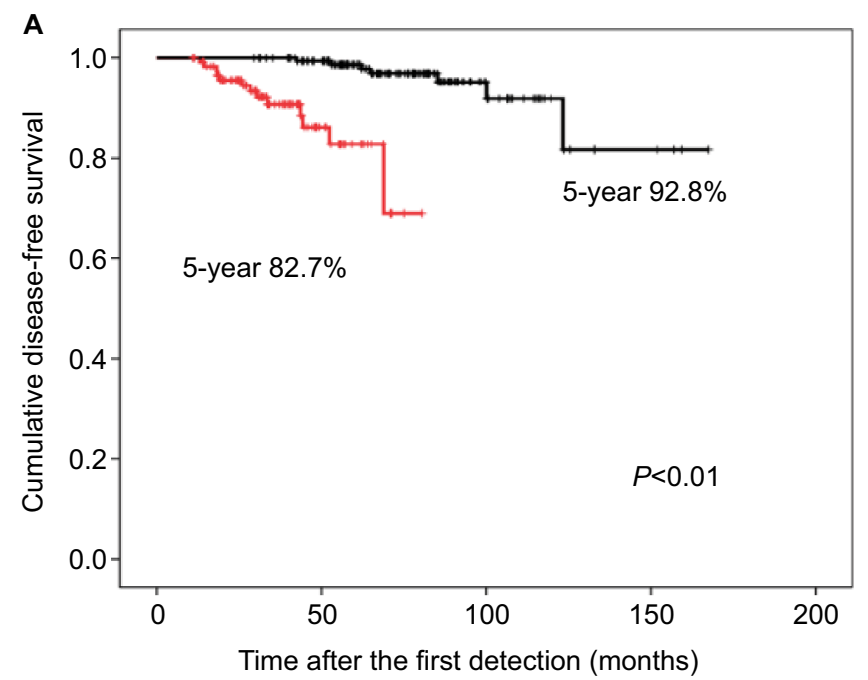

than those in the SISG $(98.7 \%$ vs $82.7 \% ; P<0.01 \%$ and $99.3 \%$ vs $93.1 \% ; P<0.01$, respectively) (Figure 3 ). Additionally, the 10-year DFS and OS rates after the first CT detection in the LISG were $92.8 \%$ and $97.3 \%$, respectively (Figure 3).

On Cox multivariate regression analyses, which incorporated the results of univariate analysis, CT consolidation (ratio $>0.5$ ), a CEA abnormality and the mutation status (triple negative) were found to be independent predictors of an unfavorable prognosis, as defined by DFS after the first CT detection (Table 2).

To study the relationship between these three factors and surgical outcomes, patients in the SISG were stratified into two subgroups: low-risk SISG (presence of 0 or one factor) $(n=81)$ and high-risk SISG (presence of a couple of factors) $(n=40)$. On Kaplan-Meier analysis, survival in the low-risk SISG was significantly longer than that in the high-risk SISG $(P<0.01$ for DFS and $P<0.01$ for OS); moreover, the survival outcomes in the low-risk SISG were comparable with those in the LISG $(P=0.76$ for DFS and $P=0.18$ for OS) (Figure 4).

\section{The relationship among the significant prognostic factors}

The presence of potential NSCLC opacities in combination with two of the three factors (CT consolidation [ratio $>0.5$ ], CEA abnormality and a triple-negative mutation status) was associated with an unfavorable prognosis. Therefore, we examined whether the mutation status was associated with CT consolidation (ratio $>0.5$ ) and CEA abnormality. We could not

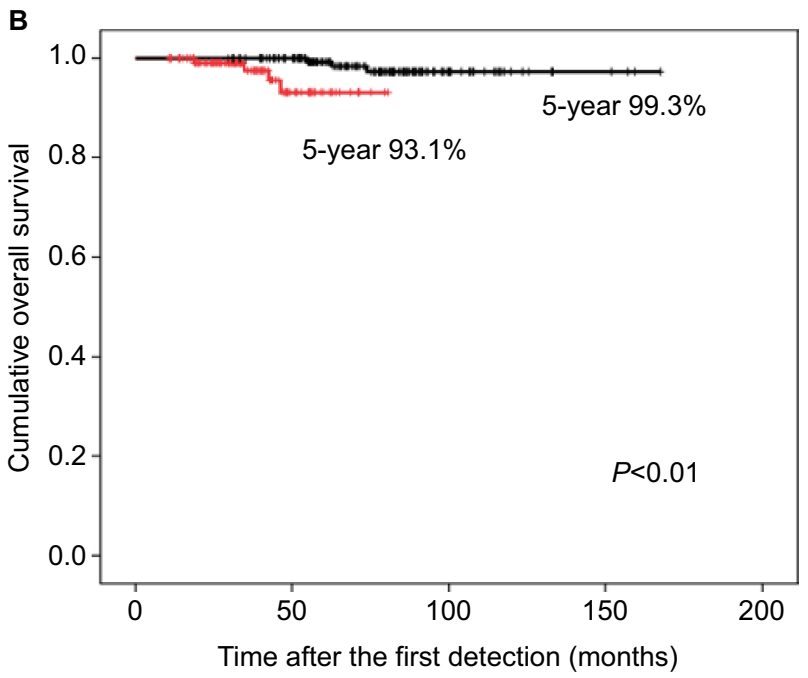

Figure 3 Kaplan-Meier graphs of overall survival in the study population.

Notes: (A) Disease-free survival after the first detection of potential non-small-cell lung cancer opacities in the study population. Black line, the LISG ( $\mathrm{n}=\mathrm{I72}$ ) and red line, the SISG $(n=121)$. (B) Overall survival at the first detection. Black line, the LISG $(n=172)$ and red line, the SISG $(n=121) * P<0.05$.

Abbreviations: LISG, longer-interval surgery group; SISG, shorter-interval surgical group. 
Table I Characteristics of patients

\begin{tabular}{|c|c|c|c|}
\hline Variables & SISG $(N=|2|)$ & DSG (N=172) & $P$-value \\
\hline Age (years), median (range) & $67(37-86)$ & $67(32-86)$ & 0.82 \\
\hline Gender (male/female), n & $69 / 52$ & $83 / 89$ & 0.14 \\
\hline Tobacco (pack-year), mean \pm SD & $29.3 \pm 35.1$ & $20.3 \pm 31.6$ & $0.02 *$ \\
\hline CEA $(\mathrm{ng} / \mathrm{mL}$, mean $\pm \mathrm{SD})$ & $3.3 \pm 3.6$ & $3.3 \pm 4.5$ & 0.19 \\
\hline \multicolumn{4}{|l|}{$\mathrm{CT}$ findings, mean $\pm \mathrm{SD}$} \\
\hline CT consolidation ratio (\%) & $67.6 \pm 30.8$ & $61.4 \pm 30.3$ & 0.08 \\
\hline Mediastinal diameter $(\mathrm{mm})$ & $10.2 \pm 8.2$ & $7.9 \pm 7.3$ & $0.02 *$ \\
\hline Tumor size $(\mathrm{mm})$ & $20.1 \pm 7.8$ & $18.9 \pm 7.2$ & 0.24 \\
\hline Doubling tumoral time (months) & $155.8 \pm 317.2$ & $405.7 \pm 902.4$ & $<0.0 I^{*}$ \\
\hline SUV max on PET, mean \pm SD & $5.8 \pm 4.5$ & $4.5 \pm 4.4$ & 0.10 \\
\hline History of malignancy, yes, n (\%) & $45(37.2)$ & $95(55.2)$ & $<0.0 I^{*}$ \\
\hline Lung cancer, $\mathrm{n}$ & 14 & 27 & \\
\hline Others, $\mathrm{n}$ & 31 & 68 & \\
\hline Open thoracotomy/TS, n & $27 / 94$ & $33 / 139$ & 0.51 \\
\hline Procedures, sublobar, n (\%) & $67(55.3)$ & $94(54.7)$ & 0.90 \\
\hline Wedge resection, $\mathrm{n}$ & 34 & 51 & \\
\hline Segmentectomy, n & 33 & 43 & \\
\hline Lobectomy, n & 54 & 78 & \\
\hline Histology (AIS + MIA, \%), n & 24.0 & 33.7 & 0.07 \\
\hline $\mathrm{AIS}+\mathrm{MIA}, \mathrm{n}$ & 29 & 58 & \\
\hline Invasive adenocarcinoma, $\mathrm{n}$ & 71 & 105 & \\
\hline Squamous, $n$ & 18 & 7 & \\
\hline Others, $\mathrm{n}$ & 3 & 2 & \\
\hline Pathological stage, more than pIB, n (\%) & $25(20.7)$ & $16(9.3)$ & $<0.0 I^{*}$ \\
\hline $0 / I A, n$ & $7 / 89$ & $18 / 138$ & \\
\hline IB/IIA/IIB/III/IV, n & $|5 / 5 / 1 / 3 /|$ & $10 / 2 / 1 / 3 / 0$ & \\
\hline Any mutations, yes, n (\%) & 71 (58.7) & $112(65.1)$ & 0.26 \\
\hline EGFR/ALK/KRAS, $\mathrm{n}$ & $52 / 17 / 2$ & $85 / 22 / 5$ & \\
\hline Triple negative, $\mathrm{n}$ & 50 & 60 & \\
\hline
\end{tabular}

Note: $* P<0.05$.

Abbreviations: AIS, adenocarcinoma in situ; CEA, carcinoembryonic antigen; CT, computed tomography; DSG, delayed surgery group; MIA, minimally invasive adenocarcinoma; PET, positron emission tomography; SISG, shorter-interval surgery group; SUV, standardized uptake value; TS, thoracoscopic surgery.

Table 2 Results of univariate and multivariate analyses for disease-free survival after the first detection of potential NSCLC opacities

\begin{tabular}{|c|c|c|c|}
\hline & \multirow{2}{*}{$\begin{array}{l}\text { Univariate } \\
P \text {-value }\end{array}$} & \multicolumn{2}{|l|}{ Multivariate } \\
\hline & & HR (95\% Cl) & $P$-value \\
\hline Age & 0.25 & & \\
\hline Gender (female) & $0.02 *$ & $0.49(0.15-1.59)$ & 0.24 \\
\hline Smoking (pack-year >40) & 0.11 & $0.76(0.25-2.26)$ & 0.62 \\
\hline History of various cancer (yes) & 0.79 & & \\
\hline \multicolumn{4}{|l|}{ Tumor marker } \\
\hline CEA abnormality $(>5 \mathrm{ng} / \mathrm{mL})$ & $<0.01 *$ & $3.11(1.25-7.73)$ & $0.02 *$ \\
\hline \multicolumn{4}{|l|}{ CT findings } \\
\hline CT consolidation ratio $(>0.5)$ & $<0.01 *$ & $7.49(1.47-38.2)$ & $0.02 *$ \\
\hline Mediastinal diameter $(>5 \mathrm{~mm})$ & $<0.01 *$ & $1.82(0.68-4.88)$ & 0.24 \\
\hline Tumor size $(>10 \mathrm{~mm})$ & 0.75 & & \\
\hline Sublobar resection (yes) & 0.37 & & \\
\hline Mutation status (EGFR/ALK/KRAS) & $0.02 *$ & $0.30(0.1 \mathrm{I}-0.83)$ & $0.02 *$ \\
\hline
\end{tabular}

Note: $* P<0.05$.

Abbreviations: CEA, carcinoembryonic antigen; CT, computed tomography; NSCLC, non-small-cell lung cancer. 
completely predict the mutation status based on preoperative clinical characteristics. Among patients with EGFR mutation, the proportion of those with CT consolidation (ratio $>0.5$ ) and CEA abnormality was significantly lower than that in patients without EGFR mutation. ALK showed significantly higher in CT consolidation (ratio $>0.5$ ) and triple-negative mutation showed higher in CEA abnormality (Table 3).

\section{Discussion}

The present study was designed to determine the impact of follow-up intervals between the time of the first CT detection of potential NSCLC opacities and eventual pulmonary resection. In addition, we conducted a retrospective analysis of clinicopathological variables that may influence patient prognosis in the same period. The results of this study can be summarized as follows: 1) by monitoring patients accord-

A

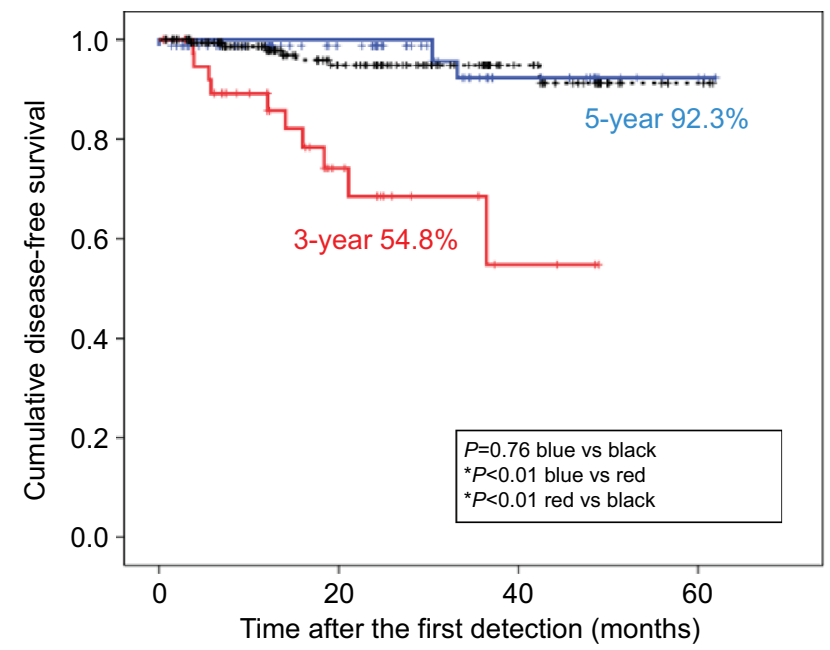

ing to protocol, a prognostic disadvantage was determined in those followed-up for $\leq 2$ years; 2) a demonstrable CT consolidation (ratio $>0.5$ ), a CEA abnormality and a triplenegative mutation at detection conferred a worse prognosis; and (c) in some members of the SISG, it would not have been beneficial to forego any further surgical delay. Some prospective follow-up studies have assessed the natural history of GGOs; however, we found no definitive evidence to identify a particular time point at which resorting to pulmonary resection (rather than continued observation) would accrue benefit. Moreover, obtaining a pathological diagnosis during a relatively long-term follow-up should help to determine the nature of the GGO.

Clinical management of potential NSCLC opacities can be challenging, given that overdiagnosis has always been a problem. ${ }^{11,12}$ If the solid component on CT is used to gage

B

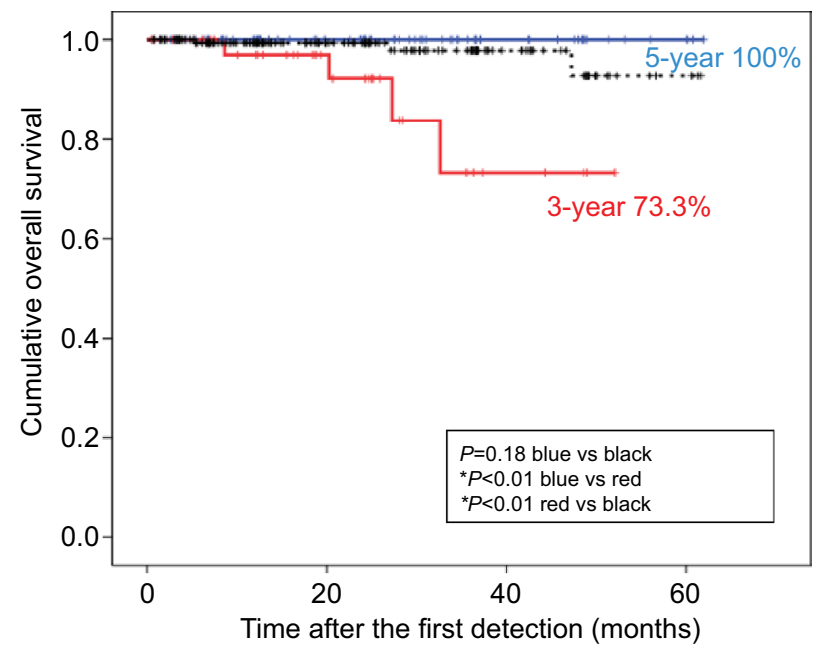

Figure 4 Kaplan-Meier graphs of overall survival in the SISG.

Notes: (A) Disease-free survival after the first detection of potential NSCLC opacities in all enrolled patients. Black line: the LISG ( $n=172$ ); blue line: the low-risk SISG $(n=8 I)$; and red line: the high-risk SISG $(n=40)$. (B) Overall survival at the first detection. Black line: the LISG ( $n=172)$; blue line: the low-risk SISG ( $n=81)$; and red line: the high-risk SISG $(n=40)$. *Indicates significance $(P<0.05)$.

Abbreviations: CT, computed tomography; LISG, longer-interval surgery group; NSCLC, non-small-cell lung cancer; SISG, shorter-interval surgical group.

Table 3 The relationship among the mutation status and the other prognostic factors

\begin{tabular}{|c|c|c|c|}
\hline \multicolumn{4}{|l|}{ Mutation status } \\
\hline CT consolidation (ratio) & CT consolidation ratio $0-0.5(n=163)$ & CT consolidation ratio $>0.5(n=130)$ & $P$-value \\
\hline EGFR & $88(54.0 \%)$ & $49(37.7 \%)$ & $<0.0 I^{*}$ \\
\hline KRAS & $18(11.0 \%)$ & $21(16.2 \%)$ & 0.20 \\
\hline$A L K$ & $\mathrm{I}(0.6 \%)$ & $6(4.6 \%)$ & $0.03^{*}$ \\
\hline Triple negative & $56(34.4 \%)$ & $54(4 \mid .5 \%)$ & 0.21 \\
\hline CEA & CEA within normal limit ( $\leq 5 \mathrm{ng} / \mathrm{mL}),(\mathrm{n}=257)$ & CEA abnormality (>0.5 ng/mL), $\mathrm{n}=36$ ) & $P$-value \\
\hline EGFR & $126(49.0 \%)$ & II (30.6\%) & $0.04^{*}$ \\
\hline KRAS & $36(14.0 \%)$ & $3(8.3 \%)$ & 0.35 \\
\hline ALK & $6(2.3 \%)$ & $\mathrm{I}(2.8 \%)$ & 0.87 \\
\hline Triple negative & 89 (34.6\%) & $21(58.3 \%)$ & $<0.01 *$ \\
\hline
\end{tabular}

Note: $* P<0.05$.

Abbreviations: CEA, carcinoembryonic antigen; CT, computed tomography. 
surgical indication, it may not retain a pathological IA stage, as observed in three prospective follow-up studies. ${ }^{5,6,13-16}$ In a recent prospective study in Japan, out of the 1,229 patients with subsolid nodules, 91 (7.4\%) patients underwent resection over a mean follow-up duration of 4.3 years. Pathological examination revealed a wide array of lesions in these 91 patients (cIA, 12; MIA, 40; AIS, 33; and atypical adenomatous hyperplasia, 6); 78 patients were found to have stage IA disease, while only 2 patients had stage IB disease. ${ }^{13}$ In the Netherlands Leuvens Longkanker Screenings Onderzoek trial, 33 out of the 108 $(28 \%)$ patients with subsolid nodules underwent surgical resection; of these, only 19 patients were found to have invasive cancer. ${ }^{14}$ Histologically confirmed lesions were stage IA $(n=18)$ or IB $(n=2)$, and one was stage IV. ${ }^{14}$ Unfortunately, the mean duration of follow-up of patients who underwent resection was not reported. Diagnostic testing in the IELCAP study $(\mathrm{N}=2,325)$ led to the discovery of lung cancer in 79 part-solid nodules, with a median time of 6 months from baseline CT to treatment. ${ }^{3}$ Histological examination confirmed that 73 were invasive, including one at stage IIIB. ${ }^{3}$ Clearly, a pathological diagnosis of invasive cancer is seldom encountered in such studies, although contrary to expectations, advanced disease remains a possibility. In our cohort, $\sim 80 \%$ of patients in the SISG and more than $90 \%$ of those in the LISG were diagnosed with pathological stage IA + AIS, despite a series of obstacles such as patient refusal for treatment, equivocal radiological findings, and difficulty in distinguishing between cancer and inflammatory lesions. This study suggested that the underestimate of radiological findings was associated with unfavorable prognosis because of the significant number of pathological stage migration. A prompt consultation on the issue of pulmonary resection is imperative to avoid under- or overdiagnosis based on cursory inspection.

Several studies have shown that the slow progression and slow growth of GGOs (with a corresponding tumor doubling time of $>2$ years) are associated with a favorable prognosis. ${ }^{15,16}$ Furthermore, stability in size and internal density of tumors during 2 years of periodic CT monitoring are strongly suggestive of a benign process. ${ }^{16,17}$ In our study, although a significantly worse prognosis was evident in the SISG (vs the LISG), the OS rates in SISG members without CT consolidation (ratio $>0.5$ ) were comparable to that in the LISG. The radiological indices cited herein, namely, CT consolidation (ratio $>0.5$ ), may well play a key role in this setting; however, if consolidation on CT is vague, which hampers surgical decision making, MD on mediastinal setting on CT is a practical and useful substitute. It is widely known that CT consolidation is strongly predictive of diminished survival in patients with NSCLC. ${ }^{11,12,18}$ In a secondary analysis of data from the NLST, Yip et al reported that NSCLC-related deaths, which occurred in 48 participants (1.9\%), were most likely attributable to solid or part-solid dominant nodules, rather than to nonsolid lesions. ${ }^{7} \mathrm{CT}$ consolidation is usually excess of MD; however, if these CT criteria are met at the first detection or during a follow-up examination, early diagnostic efforts are advisable. With such an approach, individuals who are ill-suited for conservative management may benefit from timely surgical intervention. Still, in clinical practice, thoracic physicians may sometimes struggle to differentiate primary lung cancer from inflammatory lesions. Our findings should facilitate such distinctions, help to avoid misjudgment of malignant transformation, and facilitate appropriate surgical decision-making without hesitation.

A second issue is that pathological upstaging was disproportionately higher in the SISG (vs the LISG). Since 2004, we have investigated the relationship between MD and pathological features or a postoperative prognosis. ${ }^{11,12,19}$ In a prior cohort, we showed that invasive size roughly approximated $\mathrm{MD}+3 \mathrm{~mm}$ and that was best predicted by MD. ${ }^{12}$ Nakinuma et al repeatedly found in separate cohorts that the cut-off value for differentiating AIS and MIA from IA was a maximal diameter of $3-3.3 \mathrm{~mm}$, as seen via a mediastinal window. ${ }^{13,17}$ However, in this study, we found no evidence to support the notion that $\mathrm{MD}<5 \mathrm{~mm}$ at the first CT detection of a potential NSCLC opacities should be a strong impetus to terminate observation in favor of surgery. In addition, we have previously reported that the incidence of lymph node metastases varied in accordance with MD (10 mm, $0 \%$; $10-15 \mathrm{~mm}, 34.6 \%$ ) in 176 small adenocarcinomas. ${ }^{18}$ Thus, there may be a heightened potential for tumor upstaging at $\mathrm{MD}>10 \mathrm{~mm}$. Patients who showed no radiological risk factors (ie, CT consolidation ratio $\leq 0.5$ and a favorable $\mathrm{MD}[\leq 5$ $\mathrm{mm}]$ ) were followed-up as per the recommendations, whereas patients with demonstrable radiological risk proceeded to further intervention.

In this study, CT consolidation (ratio $>0.5$ ) showed a statistically stronger relationship with $A L K$ and a weaker relationship with $E G F R$ mutation, which is consistent with our previous reports. ${ }^{20} \mathrm{~A}$ pathologically solid predominant and invasive tumor component and a CEA abnormality are known to be unfavorable postsurgical prognostic factors in NSCLC. In addition, we have conducted studies to improve the approximate accuracy of preoperative simulation of the pathologically invasive component. ${ }^{11,12,18,19}$ Recently, we demonstrated that solid-predominant tumors had the highest expression among the various histological subtypes; however, the number of genomic triple-negative adenocarcinomas was not significantly higher than that of adenocarcinomas harboring an exclusive gene of $E G F R, K R A S$, or $A L K$ on analyses 
of 324 cIA adenocarcinomas using positron emission tomography and three-dimensional CT volumetry. ${ }^{11}$ Correlations between invasive components, intratumoral heterogeneity, and mutation status should be explored in further studies.

\section{Limitations}

This study has several limitations. First, the follow-up periods and surgical indications were not integrated. When arranging various follow-up periods in patients with probable lung cancer, there is a hospital orientation for treatment purposes; the entire follow-up schedule in this study was similar to those in other studies. ${ }^{3,13,14}$ On confirmation of the patient's intention, we opt for further observation or prompt treatment (including surgery), if necessary. However, a definitive surgical strategy for potential NSCLC opacities has not yet been established. The Japanese Society of CT Screening recommended that surgical intervention strategies should depend on the lesion size ( $>15 \mathrm{~mm}$ ) or the solid component. ${ }^{21}$ Therefore, a definitive strategy that specifies an optimal time for surgical intervention awaits further studies. Another issue is the retrospective design and the single-center scope of our study. Although our study population were relatively large, an inherent potential bias for surgical intervention cannot be ruled out. In addition, MD may have been overestimated, and measurement errors may affect the assessment of CT consolidation. Finally, the scope of our study did not include benign tumors; therefore, our findings may not be applicable to the distinction between benign and malignant neoplasms.

\section{Conclusion}

In summary, this study showed that in patients with potential NSCLC opacities who are followed-up for $\leq 2$ years prior to pulmonary resection, an unfavorable prognosis is more likely, although the level of difficulty in ascertaining malignancy may also be a factor. Our recommendation is to abandon plans of patient observation in favor of surgery if CT images show either a consolidation ratio $>0.5$ or CEA abnormality.

\section{Disclosure}

The authors report no conflicts of interest in this work.

\section{References}

1. Bak SH, Lee HY, Kim JH, et al. Quantitative CT scanning analysis of pure ground-glass opacity nodules predicts further CT scanning change. Chest. 2016;149(1):180-191.

2. Gandara DR, Aberle D, Lau D, et al. Radiographic imaging of bronchioloalveolar carcinoma: screening, patterns of presentation and response assessment. J Thorac Oncol. 2006;1(9 Suppl):S20-S26.
3. Henschke CI, Yankelevitz DF, Mirtcheva R, et al. CT screening for lung cancer: frequency and significance of part-solid and nonsolid nodules. AJR Am J Roentgenol. 2002;178(5):1053-1057.

4. Hasegawa M, Sone S, Takashima S, et al. Growth rate of small lung cancers detected on mass CT screening. Br J Radiol. 2000;73(876): 1252-1259.

5. Patz EF, Caporaso NE, Dubinett SM, et al. National Lung Cancer Screening Trial American College of Radiology Imaging Network Specimen Biorepository originating from the Contemporary Screening for the Detection of Lung Cancer Trial (NLST, ACRIN 6654): design, intent, and availability of specimens for validation of lung cancer biomarkers. J Thorac Oncol. 2010;5(10):1502-1506.

6. National Lung Screening Trial Research Team, Aberle DR, Berg CD, et al. The National Lung Screening Trial: overview and study design. Radiology. 2011;258(1):243-253.

7. Yip R, Yankelevitz DF, Hu M, et al. Lung cancer deaths in the National Lung Screening Trial attributed to nonsolid nodules. Radiology. 2016;281(2):589-596.

8. Yano M, Yoshida J, Koike T, et al. Survival of 1737 lobectomy-tolerable patients who underwent limited resection for cStage IA non-small-cell lung cancer. Eur J Cardiothorac Surg. 2015;47(1):135-142.

9. Travis WD, Asamura H, Bankier AA, et al. The IASLC Lung Cancer Staging Project: Proposals for Coding T Categories for Subsolid Nodules and Assessment of Tumor Size in Part-Solid Tumors in the Forthcoming Eighth Edition of the TNM Classification of Lung Cancer. J Thorac Oncol. 2016;11(8):1204-1223.

10. Beasley MB, Brambilla E, Travis WD. The 2004 World Health Organization classification of lung tumors. Semin Roentgenol. 2005 40(2): 90-97.

11. Kuroda H, Mori S, Tanaka H, et al. Prognostic significance of combined radiologic imaging modalities for prognosis of clinical IA adenocarcinomas. Oncotarget. 2018;9(12):10745-10753.

12. Sakakura N, Inaba Y, Yatabe Y, et al. Estimation of the pathological invasive size of pulmonary adenocarcinoma using high-resolution computed tomography of the chest: a consideration based on lung and mediastinal window settings. Lung Cancer. 2016;95:51-56.

13. Kakinuma R, Noguchi M, Ashizawa K, et al. Natural history of pulmonary subsolid nodules: a prospective multicenter study. JThorac Oncol. 2016;11(7):1012-1028.

14. Scholten ET, de Jong PA, de Hoop B, et al. Towards a close computed tomography monitoring approach for screen detected subsolid pulmonary nodules? Eur Respir J. 2015;45(3):765-773.

15. Lee SW, Leem CS, Kim TJ, et al. The long-term course of ground-glass opacities detected on thin-section computed tomography. Respir Med. 2013;107(6):904-910.

16. Nakata M, Saeki H, Takata I, et al. Focal ground-glass opacity detected by low-dose helical CT. Chest. 2002;121(5):1464-1467.

17. Kakinuma R, Ohmatsu H, Kaneko M, et al. Progression of focal pure ground-glass opacity detected by low-dose helical computed tomography screening for lung cancer. J Comput Assist Tomogr. 2004;28(1): $17-23$.

18. Chang B, Hwang JH, Choi YH, et al. Natural history of pure groundglass opacity lung nodules detected by low-dose CT scan. Chest. 2013;143(1):172-178.

19. Sakao Y, Nakazono T, Tomimitsu S, et al. Lung adenocarcinoma can be subtyped according to tumor dimension by computed tomography mediastinal-window setting. Additional size criteria for clinical T1 adenocarcinoma. Eur J Cardiothorac Surg. 2004;26(6):1211-1215.

20. Nakada T, Okumura S, Kuroda H, et al. Imaging characteristics in ALK fusion-positive lung adenocarcinomas by using HRCT. Ann Thorac Cardiovasc Surg. 2015;21(2):102-108.

21. The Japanese Society of CT Screening. Online Resources: Guidelines for the Management of Pulmonary Nodules Detected by low-dose CT Lung Cancer Screening; Version 3. Available from: http://cir.ncc.go.jp/ jp/jmct3.html. Accessed December 9, 2018. 
Cancer Management and Research

\section{Publish your work in this journal}

Cancer Management and Research is an international, peer-reviewed open access journal focusing on cancer research and the optimal use of preventative and integrated treatment interventions to achieve improved outcomes, enhanced survival and quality of life for the cancer patient.

The manuscript management system is completely online and includes

Submit your manuscript here: https://www.dovepress.com/cancer-management-and-research-journal 